\title{
Vegetative propagation of Karanja (Pongamia pinnata L. Pierre ) through stem cuttings
}

\author{
S. Rout ${ }^{1^{*}}$ and S. Nayak ${ }^{2}$ \\ ${ }^{1}$ School of Forestry \& Environment, Sam Higginbottom Institute of Agriculture Technology and Sciences (Deemed \\ -to-be-University), Allahabad-211007 (Uttar Pradesh), INDIA. \\ ${ }^{2}$ Department of Forest Products and Utilization, College of Forestry, Orissa University of Agriculture and Technol- \\ ogy, Bhubaneswar-751003 (Odisha), INDIA. \\ *Corresponding author. E-mail: srout.forestry@gmail.com
}

Received: February 11, 2015; Revised received: August 22, 2015; Accepted: October 21, 2015

\begin{abstract}
The present study on vegetative propagation of Karanja ( Pongamia pinnata L.Pierre ) through stem cuttings showed maximum sprouting $(100 \%)$, rooting $(90 \%)$, rooting length $(28.33 \mathrm{~cm})$, root number $(24.66)$, fresh biomass $(63.66 \mathrm{~g})$, dry biomass $(34.38 \mathrm{~g})$ in semi hard wood cutting of $25 \mathrm{~cm}$ length treated with $1000 \mathrm{ppm}$ IAA, hard wood cutting of $25 \mathrm{~cm}$ length treated with $800 \mathrm{ppm}$ IBA, hard wood cuttings of $15 \mathrm{~cm}$ length treated with $1000 \mathrm{ppm}$ IAA, hard wood cutting of $25 \mathrm{~cm}$ length treated with $2000 \mathrm{ppm}$ IAA, semi hard wood cuttings of $25 \mathrm{~cm}$ length treated with $200 \mathrm{ppm}$ IBA, and hard wood cutting of $25 \mathrm{~cm}$ length treated with $1000 \mathrm{ppm}$ IAA, respectively. It showed that hard wood cuttings of $25 \mathrm{~cm}$ length treated with IBA promotes better sprouting, rooting percent, root number and biomass than IAA. For production of healthy seedlings the semi hard wood cutting of $25 \mathrm{~cm}$ length should be treated with 200 ppm IBA.
\end{abstract}

Keywords: Cuttings, IAA, IBA, Pongamia pinnata

\section{INTRODUCTION}

India ranks sixth in terms of energy demand. Its economics is projected to grow at $8-9 \%$ over next two decades and its energy demand is accepted to grow at an annual rate of $4-8 \%$ over the next couple of decades. So "Energy independence" will be one of the vital areas to make India developed nation (Sahoo et.al., 2014). Among the bioenergy, plants role is considered very promising because of its renewable nature and sustained production with low cost maintenance. Biodiesel produced from trees yielding oil is fast emerging as a viable alternative to petro diesel, particularly in the fall of its diminishing supply and resulting steep increase in price because of heavy requirement of edible oil, non edible oil yielding plants and trees are considered ideal for Indian condition for the production of biodiesel. India has many non-edible tree borne oil seed of which $P$. pinnata has a lot of potential. $P$. pinnata locally known as Karanja is an indigenous tree to India. It is a medium sized evergreen or briefly deciduous glabrous tree, $15-25 \mathrm{~m}$ high, with straight or crooked trunk $50-80 \mathrm{~cm}$ or more diameter and broad crown of spreading or drooping branches. The bark is greyish green or brown, smooth or covered with tubercles, leaves compound, imparipinnate, leaflets opposite, 5-9 in number ovate or elliptic. Flowers white tinged with pink or violet, fragrant, in axillary racemes pods are compressed woody, elliptic to obliquely oblong pointed at both ends, Indehiscent, yellowish gray when ripe, varying in size and shape, $4.0-7.5 \mathrm{~cm}$ long and $1.7-3.2 \mathrm{~cm}$ broad, seeds usually one, rarely two, elliptical or reniform, $1.7-2.0 \mathrm{~cm}$ long and $1.2-1.8 \mathrm{~cm}$ broad, wrinkled with reddish brown leathery testa. It is found almost throughout India up to an altitude of $1200 \mathrm{~m}$ (Chaturvedi, 1975). It is chiefly found along the bank of stream and river or near sea coast, beach and tidal forest (Troup, 1921). It is widely grown from tropical dry to subtropical dry forest. It is shade bearer and is considered to be good tree for planting in pastures, as grasses grow well in its shade. The tree is suitable for afforestation especially in watershed areas and drier parts of country. Andra Pradesh, Haryana, Karnataka, Madhya Pradesh, Odisha, Rajasthan, Tamil Nadu and Uttar Pradesh are the potential states in the country. Large numbers of Karanja trees have also been planted in roadside both in highways and also in urban area during last two decades. The yield of fruits varies from 9 to $90 \mathrm{~kg}$ per tree for different age trees (CSIR, 1966). The oil yield is reported to be about $32 \%$ ( Rahman, 1977). Besides its use for production of bio-diesel the oil is also used for tanning leather, soap, as illuminating oil and for lubrication. The oil cake is used as pollutary feed ( Mandal and Banarjee, 1982). The oil is also used for curing rheumatism, Powdered seed is used as febrifuge, tonic and for curing bronchitis and whooping cough. Flowers are used for diabetics ( Hewamanna et 
al., 2004) and bark for internal bleeding piles, diarrhea (Brijesh et al., 2006) and curing beriberi. Karanja can be successfully propagated through seeds and cuttings. Plants propagated through seeds are heterozygous, also delay the flowering, fruiting and seed production where as in vegetatively propagated seedlings are one of the superior traits of the mother plants with respect to yield, oil content and disease resistant are maintained and thereby reducing the seed bearing age. Keeping in view of the importance of the crop and its propagation methods, the present experiment was designed with the objective of mass production of quality planting material through vegetative propagation of $P$. pinnata.

\section{MATERIALS AND METHODS}

The investigation was carried out at College of Forestry, Orissa University of Agriculture and Technology (OUAT), Bhubaneswar during February, 2012. The experiment was started with collection of hardwood, semi hardwood and soft wood cutting of $P$. pinnata from identified plus trees. The cutting of selected healthy branches bring out which were of $15 \mathrm{~cm}$ and $25 \mathrm{~cm}$ lengths having 4 to 5 nodes under each category of soft wood (with in thickness $<0.5$ ), semi hard wood $(0.5-0.7)$ and hard wood $(1.5$ to $2 \mathrm{~cm})$. The bases of hardwood, semi hardwood and soft wood portions of cuttings were dipped in the 200,400, 800, 1000 and 2000 ppm of IAA and IBA respectively for four hours. After which the cutting were planted in the polypots filled with rooting media consists of sand, soil and farm yard manure (FYM) in ratio of $1: 2: 1$. The polypots were put under agro-shed net and watered as per the requirement. Observations like sprouting percentage, rooting percentage, mean root length $(\mathrm{cm})$, mean number of roots, mean fresh biomass $(\mathrm{g})$ and mean dry biomass (g) were observed following standard methods (Zerhoune, 2003; Sahoo et.al., 2014). The experiment was designed in completely randomized design (CRD), replicated thrice and data observed were subjected to statistical analysis as for the methods detailed by Gomez and Gomez (1984). The data were transferred from where ever required before suitability of Analysis of Variance (ANOVA) analyzed in statistical package SAS version 7.0.

\section{RESULTS AND DISCUSSION}

The result obtained during the present course of investigation was carried out to visualize a significant influence of IAA and IBA on the cuttings of $P$. pinnata. Maximum sprouting was recorded in semi hard wood cuttings of $25 \mathrm{~cm}$ length treated with 1000 ppm IAA $(100 \%)$ and minimum was recorded in soft wood cuttings of $25 \mathrm{~cm}$ length with $400 \mathrm{ppm}$ of IBA (63.33\%) (Table 1). Earliness in sprouting and increase in number of sprouts may be due to better utilization of stored carbohydrates, nitrogen and other factors with the help of growth regulators (Chandramouli, 2001; Sahoo et.al., 2014).Further stored food materials with the help of growth regulators have fastened the sprouting there by enhancing the utilization of carbohydrates at base of cuttings through photosynthesis (Haissig, 1984). According to Wright, (1975) that vegetative propagation, the sprouting depends on food reserve available within the cuttings. In case of rooting percentage (Table 2) among IAA and IBA effect on rooting of different types and length of cuttings of $P$. pinnata, IBA treated in hard wood cuttings of $25 \mathrm{~cm}$ length showed maximum rooting $(60 \%)$ for the mean value of rooting percentage (over all concentration) where as soft wood cuttings of $15 \mathrm{~cm}$ length treated with IBA resulted in minimum rooting $(18.88 \%)$. The effect may be due to the slow translocation property or slow destruction by auxin destroying enzyme system ( Debnath and Maiti,1990). It is also came to know from this investigation that IBA treated $25 \mathrm{~cm}$ length hard wood cuttings at the concentration of $800 \mathrm{ppm}$ gave the maximum rooting of $P$. pinnata $(90 \%)$ compared to with control treated in distil water of $15 \mathrm{~cm}$ length of soft wood cuttings which is minimum $(0 \%)$.This may be due to better response to optimum concentration of IBA may be attribute to increase rate of respiration, accumulation of higher level of amino acids of base after 4 hours the treatment with auxins than in control treated with distil water. This pattern is continued with nitrogenous substances accumulating in the basal part of treated cuttings, apparently mobilized in the upper part and translocated as aspergene (Strydom and Hartmann, 1960). Palanisamy and Kumar, (1997) reported that exogenously applied auxins are sensitive to activate the cambium probably in the active period of cambium resulting significant root formation and also mentioned that IBA induced more number of roots during the months of January, February, March and April and 800 ppm IBA proved to be most suitable for adventitious rooting and formation of more number of roots in case of $P$. pinnata. The relatively poor rooting with IAA treated stem cuttings of $P$. pinnata in comparison to IBA could be explained by the sensitivity of IAA to light (Hartmann et al. 2002), production of more ethylene which is known to inhibit the root production (Mullins, 1972 ) and could also be due to the higher metabolic turnover. IBA is less sensitive than IAA to non biological degradation such as photo oxidation (Epstein and Ludwig-Muller, 1993; De Klerk et al. 1997) .In general IBA 800 ppm has been found to induce better root system in $P$. pinnata cuttings. The basis for this may be enhancement of hydrolysis of nutrient response (mainly starch) by auxin treatment .According to Nanda et al., (1968) enhanced hydrolysis activity in the presence of exogenously applied hormones was responsible for the increased rooting in auxin treated cuttings. IBA treatment enhanced the cambial activity (Davies et al., 1982; Davies, 1984). In case of soft wood, semi hard wood, hard wood cuttings of $15 \mathrm{~cm}$ length treated with 
Table 1. Effect of IAA and IBA on sprouting (\%) of different types of cuttings of $P$. pinnata.

\begin{tabular}{|c|c|c|c|c|c|c|c|c|}
\hline S.N. & Cutting Type & $\begin{array}{c}200 \\
\mathrm{ppm}\end{array}$ & $\begin{array}{c}400 \\
\mathrm{ppm}\end{array}$ & $\begin{array}{c}800 \\
\text { ppm }\end{array}$ & $\begin{array}{l}1000 \\
\text { ppm }\end{array}$ & $\begin{array}{l}2000 \\
\mathrm{ppm}\end{array}$ & Control & Mean \\
\hline 1. & $\begin{array}{l}15 \mathrm{~cm} \text { soft wood cut- } \\
\text { ting + IAA }\end{array}$ & $\begin{array}{c}73.33 \\
(58.89)\end{array}$ & $\begin{array}{c}83.33 \\
(65.88)\end{array}$ & $\begin{array}{c}73.33 \\
(58.89)\end{array}$ & $\begin{array}{c}83.33 \\
(65.88)\end{array}$ & $\begin{array}{c}86.66 \\
(68.53)\end{array}$ & $\begin{array}{c}83.33 \\
(65.88)\end{array}$ & $\begin{array}{c}80.55 \\
(63.79)\end{array}$ \\
\hline 2. & $\begin{array}{l}15 \mathrm{~cm} \text { semi hard wood } \\
\text { Cutting +IAA }\end{array}$ & $\begin{array}{l}90.00 \\
(71.56)\end{array}$ & $\begin{array}{c}93.33 \\
(75.00)\end{array}$ & $\begin{array}{c}86.66 \\
(68.53)\end{array}$ & $\begin{array}{c}93.33 \\
(75.00)\end{array}$ & $\begin{array}{c}93.33 \\
(75.00)\end{array}$ & $\begin{array}{c}80.00 \\
(65.44)\end{array}$ & $\begin{array}{c}89.44 \\
(71.00)\end{array}$ \\
\hline 3. & $\begin{array}{l}15 \mathrm{~cm} \text { hard wood cut- } \\
\text { ting + IAA }\end{array}$ & $\begin{array}{c}96.66 \\
(79.37)\end{array}$ & $\begin{array}{c}93.33 \\
(75.00)\end{array}$ & $\begin{array}{c}93.33 \\
(75.00)\end{array}$ & $\begin{array}{c}93.33 \\
(75.00)\end{array}$ & $\begin{array}{c}76.66 \\
(61.07)\end{array}$ & $\begin{array}{c}86.66 \\
(68.53)\end{array}$ & $\begin{array}{l}89.99 \\
(71.47)\end{array}$ \\
\hline 4. & $\begin{array}{l}15 \mathrm{~cm} \text { soft wood cut- } \\
\text { ting + IBA }\end{array}$ & $\begin{array}{c}86.66 \\
(68.55)\end{array}$ & $\begin{array}{c}90.00 \\
(71.56)\end{array}$ & $\begin{array}{c}90.00 \\
(71.56)\end{array}$ & $\begin{array}{c}86.66 \\
(68.53)\end{array}$ & $\begin{array}{c}86.66 \\
(68.53)\end{array}$ & $\begin{array}{c}83.33 \\
(65.88)\end{array}$ & $\begin{array}{c}87.22 \\
(69.04)\end{array}$ \\
\hline 5. & $\begin{array}{l}15 \mathrm{~cm} \text { semi hard wood } \\
\text { Cutting + IBA }\end{array}$ & $\begin{array}{c}76.66 \\
(61.07)\end{array}$ & $\begin{array}{l}93.33 \\
(75.00)\end{array}$ & $\begin{array}{c}86.66 \\
(68.53)\end{array}$ & $\begin{array}{c}83.33 \\
(65.88)\end{array}$ & $\begin{array}{c}86.66 \\
(68.53)\end{array}$ & $\begin{array}{c}80.00 \\
(65.44)\end{array}$ & $\begin{array}{c}84.44 \\
(66.74)\end{array}$ \\
\hline 6. & $\begin{array}{l}15 \mathrm{~cm} \text { hard wood cut- } \\
\text { ting + IBA }\end{array}$ & $\begin{array}{c}90.00 \\
(71.56)\end{array}$ & $\begin{array}{l}96.66 \\
(79.37)\end{array}$ & $\begin{array}{c}90.00 \\
(71.56)\end{array}$ & $\begin{array}{c}93.33 \\
(75.000)\end{array}$ & $\begin{array}{c}90.00 \\
(71.56)\end{array}$ & $\begin{array}{c}86.66 \\
(68.53)\end{array}$ & $\begin{array}{c}91.11 \\
(72.64)\end{array}$ \\
\hline 7. & $\begin{array}{l}25 \mathrm{~cm} \text { soft wood cut- } \\
\text { ting + IAA }\end{array}$ & $\begin{array}{l}80.00 \\
(63.44)\end{array}$ & $\begin{array}{c}90.00 \\
(71.56)\end{array}$ & $\begin{array}{c}70.00 \\
(56.79)\end{array}$ & $\begin{array}{c}86.66 \\
(68.55)\end{array}$ & $\begin{array}{c}73.33 \\
(58.59)\end{array}$ & $\begin{array}{c}80.00 \\
(65.44)\end{array}$ & $\begin{array}{c}79.99 \\
(63.36)\end{array}$ \\
\hline 8. & $\begin{array}{l}25 \mathrm{~cm} \mathrm{semi} \mathrm{hard} \mathrm{wood} \\
\text { Cutting + IAA }\end{array}$ & $\begin{array}{c}76.66 \\
(61.07)\end{array}$ & $\begin{array}{c}86.66 \\
(68.53)\end{array}$ & $\begin{array}{c}90.00 \\
(71.56)\end{array}$ & $\begin{array}{l}100.00 \\
(90.00)\end{array}$ & $\begin{array}{c}90.00 \\
(71.56)\end{array}$ & $\begin{array}{c}73.33 \\
(88.89)\end{array}$ & $\begin{array}{c}86.11 \\
(68.11)\end{array}$ \\
\hline 9. & $\begin{array}{l}25 \mathrm{~cm} \text { hard wood cut- } \\
\text { ting + IAA }\end{array}$ & $\begin{array}{c}96.66 \\
(79.37)\end{array}$ & $\begin{array}{l}96.66 \\
(79.37)\end{array}$ & $\begin{array}{c}76.66 \\
(61.07)\end{array}$ & $\begin{array}{c}73.33 \\
(58.89)\end{array}$ & $\begin{array}{c}83.33 \\
(65.88)\end{array}$ & $\begin{array}{c}86.66 \\
(90.00)\end{array}$ & $\begin{array}{c}85.55 \\
(67.62)\end{array}$ \\
\hline 10. & $\begin{array}{l}25 \mathrm{~cm} \text { soft wood cut- } \\
\text { ting + IBA }\end{array}$ & $\begin{array}{c}86.66 \\
(68.53)\end{array}$ & $\begin{array}{c}63.33 \\
(52.71)\end{array}$ & $\begin{array}{c}93.33 \\
(75.00)\end{array}$ & $\begin{array}{c}73.33 \\
(58.89)\end{array}$ & $\begin{array}{l}76.66 \\
(61.07)\end{array}$ & $\begin{array}{c}80.00 \\
(68.53)\end{array}$ & $\begin{array}{c}78.88 \\
(62.58)\end{array}$ \\
\hline 11. & $\begin{array}{l}25 \mathrm{~cm} \mathrm{semi} \mathrm{hard} \mathrm{wood} \\
\text { Cutting + IBA }\end{array}$ & $\begin{array}{c}80.00 \\
(63.44)\end{array}$ & $\begin{array}{c}80.00 \\
(65.44)\end{array}$ & $\begin{array}{c}90.00 \\
(71.56)\end{array}$ & $\begin{array}{c}86.66 \\
(68.53)\end{array}$ & $\begin{array}{c}86.66 \\
(68.53)\end{array}$ & $\begin{array}{c}73.33 \\
(88.89)\end{array}$ & $\begin{array}{c}82.77 \\
(65.42)\end{array}$ \\
\hline 12 & $\begin{array}{l}25 \mathrm{~cm} \text { hard wood cut- } \\
\text { ting + IBA }\end{array}$ & $\begin{array}{c}90.00 \\
(71.56)\end{array}$ & $\begin{array}{c}86.66 \\
(68.53)\end{array}$ & $\begin{array}{c}90.00 \\
(71.56)\end{array}$ & $\begin{array}{c}90.00 \\
(71.56)\end{array}$ & $\begin{array}{c}93.33 \\
(75.00)\end{array}$ & $\begin{array}{c}86.66 \\
(68.53)\end{array}$ & $\begin{array}{c}89.44 \\
(71.00)\end{array}$ \\
\hline & Mean & $\begin{array}{c}85.27 \\
(67.37)\end{array}$ & $\begin{array}{c}87.77 \\
(69.47)\end{array}$ & $\begin{array}{c}85.83 \\
(67.86)\end{array}$ & $\begin{array}{c}86.94 \\
(68.78)\end{array}$ & $\begin{array}{c}85.27 \\
(67.37)\end{array}$ & $\begin{array}{c}81.66 \\
(64.60)\end{array}$ & \\
\hline
\end{tabular}

Cutting type $-\mathrm{CD}$ at 5\% - 6.55 (4.32); Concentration - CD at 5\% - 4.65 (3.10); Interaction CD at 5\% - 16.13 (13.26); *Figure in parenthesis are the angular transformed values.

IAA $200 \mathrm{ppm}$ and $400 \mathrm{ppm}$ respectively resulted in maximum rooting percentage. This may be due to the synergetic effect of the total amount of indigenous auxin present in the cuttings and the exogenous auxin (IAA) applied at various concentration to the different types of cuttings enhances the cambial activity at optimum photoperiod and temperature. Wareing and Roberts, 1956; Waisel and Fahn (1965); Smith and Wareing (1972) reported that endogeneous IAA, photoperiod and temperature presumed to control the cambial activity. However in case of soft wood, semi hard wood, hard wood cuttings of $25 \mathrm{~cm}$ length treated with IAA 1000 ppm, IBA 1000 ppm and IBA 800 ppm respectively resulted in maximum rooting. Similarly for root length IBA showed maximum $(19.16 \mathrm{~cm})$ which may be due to its greater stability, transportability, ability to produce roots and consequently results in promoting root length and lower mortality in plants (Shagoo et al., 2007; Abidin and Baker, 1984). The length of longest root was observed in 400ppm IBA treated with $25 \mathrm{~cm}$ length semi hard wood cuttings and 1000 ppm IAA treated with $15 \mathrm{~cm}$ hard wood cuttings separately. In case of soft wood, semi hard wood and hard wood cuttings of $15 \mathrm{~cm}$ and $25 \mathrm{~cm}$ length treated with IAA $400 \mathrm{ppm}, 1000 \mathrm{ppm}$ and IBA $1000 \mathrm{ppm}$ and
2000 ppm, respectively resulted in maximum root length. However incase of soft wood, semi hard wood and hard wood cuttings of $25 \mathrm{~cm}$ length treated with IAA $1000 \mathrm{ppm}$ and IBA $400 \mathrm{ppm}$ and $2000 \mathrm{ppm}$ respectively resulted in maximum rooting length (Table 3 ).The possible reason for IAA and IBA showing highest root length might for the early formation of roots and more utilization of reserved food materials of the treated cuttings (Ghatnatti, 1997). As per Keseri et al., (2008) maximum root length was observed in case of cuttings treated with $2.85 \mathrm{mM}$ IAA . The differences among auxins could also be related to other factors such as higher stability and slow rate of conjugation of IBA ,so that the free IBA required to induce rooting will be available over a longer period of time than IAA (Krisantini et al., 2006). Similarly for root number 15 $\mathrm{cm}$ length soft wood cuttings treated with IBA gave maximum root numbers (16.22) over other treatments and control. (Table 4) Which may be due to less sensitivity of IBA to none biological degradation such as photo oxidatation ( Epastein and Ludurig-Muller, 1993; De Klerk et al., 1997) and comparatively less production of ethylene which helps in root production. Maximum root number (24.66) was obtained in hard wood cuttings of $25 \mathrm{~cm}$ length treated with $2000 \mathrm{ppm}$ 
Table 2. Effect of IAA and IBA on rooting (\%) of different types of cuttings of $P$. pinnata

\begin{tabular}{|c|c|c|c|c|c|c|c|c|}
\hline $\begin{array}{l}\text { S. } \\
\text { N. }\end{array}$ & Туре & $\begin{array}{r}200 \\
\text { ppm }\end{array}$ & $\begin{array}{r}400 \\
\text { ppm }\end{array}$ & $\begin{array}{c}800 \\
\text { ppm }\end{array}$ & $\begin{array}{l}1000 \\
\text { ppm }\end{array}$ & $\begin{array}{l}2000 \\
\text { ppm }\end{array}$ & $\mathrm{Cc}$ & Me \\
\hline 1. & $15 \mathrm{~cm} \mathrm{soft}$ & $\begin{array}{c}40.00 \\
(39.23)\end{array}$ & $\begin{array}{c}20.00 \\
(26.56)\end{array}$ & $\begin{array}{c}23.33 \\
(28.86)\end{array}$ & $\begin{array}{c}23.33 \\
(28.86)\end{array}$ & $\begin{array}{c}26.66 \\
(31.05)\end{array}$ & $\begin{array}{c}0.00 \\
(0.00)\end{array}$ & $\begin{array}{c}22.22 \\
(28.11)\end{array}$ \\
\hline 2. & $\begin{array}{l}15 \mathrm{~cm} \text { semi hard wood Cutting + } \\
\text { IAA }\end{array}$ & $\begin{array}{c}23.33 \\
(28.86)\end{array}$ & $\begin{array}{c}36.66 \\
(37.23)\end{array}$ & $\begin{array}{c}26.66 \\
(51.05)\end{array}$ & $\begin{array}{c}20.00 \\
(26.56)\end{array}$ & $\begin{array}{c}20.00 \\
(26.56)\end{array}$ & $\begin{array}{c}20.00 \\
(26.56)\end{array}$ & $\begin{array}{c}22.77 \\
(28.45)\end{array}$ \\
\hline 3. & $\begin{array}{l}15 \mathrm{~cm} \text { hard wood cutting }+ \\
\text { IAA }\end{array}$ & $\begin{array}{c}20.00 \\
(26.56)\end{array}$ & $\begin{array}{c}40.00 \\
(84.25)\end{array}$ & $\begin{array}{c}30.00 \\
(33.21)\end{array}$ & $\begin{array}{c}20.00 \\
(26.56)\end{array}$ & $\begin{array}{c}20.00 \\
(26.56)\end{array}$ & $\begin{array}{c}20.00 \\
(26.56)\end{array}$ & $\begin{array}{c}25.00 \\
(30.00)\end{array}$ \\
\hline 4. & $15 \mathrm{~cm}$ soft wood cutting + IBA & $\begin{array}{c}20.00 \\
(26.56)\end{array}$ & $\begin{array}{c}20.00 \\
(26.56)\end{array}$ & $\begin{array}{c}26.66 \\
(51.05)\end{array}$ & $\begin{array}{c}30.00 \\
(33.21)\end{array}$ & $\begin{array}{c}16.66 \\
(23.97)\end{array}$ & $\begin{array}{l}0.00 \\
(0.00)\end{array}$ & $\begin{array}{c}18.88 \\
(25.70)\end{array}$ \\
\hline 5 . & $\begin{array}{l}15 \mathrm{~cm} \text { semi hard wood Cutting + } \\
\text { IBA }\end{array}$ & $\begin{array}{l}23.33 \\
(28.86)\end{array}$ & $\begin{array}{c}26.66 \\
(31.05)\end{array}$ & $\begin{array}{c}26.66 \\
(31.05)\end{array}$ & $\begin{array}{l}26.66 \\
(31.05)\end{array}$ & $\begin{array}{c}30.00 \\
(32.21)\end{array}$ & $\begin{array}{r}20 \\
(26\end{array}$ & $\begin{array}{l}25.55 \\
(30.33)\end{array}$ \\
\hline 6. & $15 \mathrm{~cm}$ hard wood cutting + IBA & $\begin{array}{c}33.33 \\
(35.24)\end{array}$ & $\begin{array}{c}30.00 \\
(32.21)\end{array}$ & $\begin{array}{c}26.66 \\
(31.05)\end{array}$ & $\begin{array}{l}23.33 \\
(26.86)\end{array}$ & $\begin{array}{c}26.66 \\
(31.05)\end{array}$ & $\begin{array}{r}20 \\
(26\end{array}$ & $\begin{array}{c}26.66 \\
(31.05)\end{array}$ \\
\hline 7. & d cutting + & $\begin{array}{c}16.66 \\
(23.97)\end{array}$ & $\begin{array}{c}26.66 \\
(31.05)\end{array}$ & $\begin{array}{c}50.00 \\
(45.00)\end{array}$ & $\begin{array}{c}63.33 \\
(50.71)\end{array}$ & $\begin{array}{c}50.00 \\
(45.00)\end{array}$ & $\begin{array}{r}20 \\
(26\end{array}$ & $\begin{array}{c}37.77 \\
(37.88)\end{array}$ \\
\hline 8. & $\begin{array}{l}25 \mathrm{~cm} \text { semi hard wood Cutting } \\
+ \text { IAA }\end{array}$ & $\begin{array}{c}20.00 \\
(26.56)\end{array}$ & $\begin{array}{c}43.33 \\
(41.15)\end{array}$ & $\begin{array}{c}56.66 \\
(48.79)\end{array}$ & $\begin{array}{c}43.33 \\
(41.15)\end{array}$ & $\begin{array}{c}33.33 \\
(35.24)\end{array}$ & $\begin{array}{c}36.66 \\
(37.23)\end{array}$ & $\begin{array}{c}38.88 \\
(38.53)\end{array}$ \\
\hline 9. & $\begin{array}{l}25 \mathrm{~cm} \text { hard wood cutting }+ \\
\text { IAA }\end{array}$ & $\begin{array}{c}46.66 \\
(43.05)\end{array}$ & $\begin{array}{c}60.00 \\
(50.77)\end{array}$ & $\begin{array}{c}73.33 \\
(58.89)\end{array}$ & $\begin{array}{c}56.66 \\
(48.79)\end{array}$ & $\begin{array}{c}53.33 \\
(46.89)\end{array}$ & $\begin{array}{c}30.00 \\
(33.21)\end{array}$ & $\begin{array}{c}53.32 \\
(46.89)\end{array}$ \\
\hline 10. & $25 \mathrm{~cm}$ soft wood cutting + IBA & $\begin{array}{c}93.33 \\
(41.15)\end{array}$ & $\begin{array}{c}30.00 \\
(30.21)\end{array}$ & $\begin{array}{c}33.33 \\
(35.24)\end{array}$ & $\begin{array}{c}56.66 \\
(48.79)\end{array}$ & $\begin{array}{r}53 . \\
(46 .\end{array}$ & $\begin{array}{r}20 \\
(26\end{array}$ & $\begin{array}{c}39.44 \\
(38.88)\end{array}$ \\
\hline 11. & $\begin{array}{l}25 \mathrm{~cm} \text { semi hard wood Cutting } \\
+ \text { IBA }\end{array}$ & $\begin{array}{c}50.00 \\
(45.00)\end{array}$ & $\begin{array}{c}53.33 \\
(46.89)\end{array}$ & $\begin{array}{c}53.33 \\
(46.89)\end{array}$ & $\begin{array}{l}76.66 \\
(61.07)\end{array}$ & $\begin{array}{c}56.66 \\
(48.79)\end{array}$ & $\begin{array}{c}36.66 \\
(37.23)\end{array}$ & $\begin{array}{c}54.44 \\
(47.52)\end{array}$ \\
\hline 12 & $25 \mathrm{~cm}$ hard wood cutting + IBA & $\begin{array}{c}73.33 \\
(58.89)\end{array}$ & $\begin{array}{c}56.66 \\
(48.79)\end{array}$ & $\begin{array}{c}90.00 \\
(71.56)\end{array}$ & $\begin{array}{c}40.00 \\
(39.23)\end{array}$ & $\begin{array}{c}70.00 \\
(56.79)\end{array}$ & $\begin{array}{c}30.00 \\
(33.21)\end{array}$ & $\begin{array}{c}60.00 \\
(50.77)\end{array}$ \\
\hline & Mea & $\begin{array}{c}34.16 \\
(35.73)\end{array}$ & $\begin{array}{c}36.94 \\
(37.41)\end{array}$ & $\begin{array}{c}43.05 \\
(40.98)\end{array}$ & $\begin{array}{c}40.00 \\
(39.23)\end{array}$ & $\begin{array}{c}38.05 \\
(38.06)\end{array}$ & $\begin{array}{r}21.11 \\
(27.35\end{array}$ & \\
\hline
\end{tabular}

Cutting type - $\mathrm{CD}$ at $5 \%-7.50$ (5.92); Concentration $-\mathrm{CD}$ at $5 \%-5.30$ (3.97); Interaction $\mathrm{CD}$ at $5 \%-12.37$ (9.57); *Figure in parenthesis are the angular transformed values.

Table 3. Effect of IAA and IBA on root length $(\mathrm{cm})$ in different types of cuttings of P.pinnata.

\begin{tabular}{ccccccccc}
\hline S. & Cutting Type & $\mathbf{2 0 0}$ & $\mathbf{4 0 0}$ & $\mathbf{8 0 0}$ & $\mathbf{1 0 0 0}$ & $\mathbf{2 0 0 0}$ & Control & Mean \\
N. & & $\mathbf{p p m}$ & $\mathbf{p p m}$ & $\mathbf{p p m}$ & $\mathbf{p p m}$ & $\mathbf{p p m}$ & & \\
\hline 1. & $15 \mathrm{~cm}$ soft wood cutting + IAA & 13.00 & 8.66 & 10.33 & 10.33 & 10.33 & 0.00 & 8.77 \\
2. & $15 \mathrm{~cm}$ semi hard wood Cutting + IAA & 10.00 & 10.66 & 8.66 & 10.00 & 10.00 & 7.00 & 9.38 \\
3. & $15 \mathrm{~cm}$ hard wood cutting + IAA & 10.33 & 12.66 & 20.00 & 28.33 & 21.33 & 9.00 & 16.94 \\
4. & $15 \mathrm{~cm}$ soft wood cutting + IBA & 10.66 & 12.33 & 9.66 & 15.66 & 14.66 & 0.00 & 10.50 \\
5. & $15 \mathrm{~cm}$ semi hard wood Cutting + IBA & 8.66 & 10.33 & 10.33 & 9.33 & 10.66 & 7.00 & 9.38 \\
6. & $15 \mathrm{~cm}$ hard wood cutting + IBA & 15.66 & 14.00 & 12.00 & 15.33 & 20.33 & 9.00 & 14.38 \\
7. & $25 \mathrm{~cm}$ soft wood cutting + IAA & 10.33 & 8.66 & 10.33 & 17.66 & 16.66 & 9.00 & 12.11 \\
8. & $25 \mathrm{~cm}$ semi hard wood Cutting + IAA & 10.66 & 12.00 & 14.33 & 13.00 & 13.00 & 10.33 & 12.22 \\
9. & $25 \mathrm{~cm}$ hard wood cutting + IAA & 10.00 & 12.00 & 15.00 & 18.00 & 15.66 & 12.00 & 13.77 \\
10. & $25 \mathrm{~cm}$ soft wood cutting + IBA & 10.00 & 12.33 & 15.33 & 16.66 & 14.33 & 9.00 & 12.94 \\
11. & $25 \mathrm{~cm}$ semi hard wood Cutting + IBA & 17.00 & 28.33 & 15.33 & 25.00 & 19.00 & 10.33 & 19.16 \\
12 & $25 \mathrm{~cm}$ hard wood cutting + IBA & 10.00 & 12.00 & 13.33 & 18.33 & 20.66 & 12.00 & 14.38 \\
\hline & Mean & 11.36 & 12.83 & 12.88 & 16.47 & 15.55 & 7.58 \\
\hline
\end{tabular}

Cutting type - CD at 5\% - 0.77; Concentration - CD at 5\% -0.55 ; Interaction $\mathrm{CD}$ at $5 \%-1.90$ 
Table 4. Effect of IAA and IBA on root number of different types of cuttings of $P$. pinnata.

\begin{tabular}{|c|c|c|c|c|c|c|c|c|}
\hline S.N. & Cutting Type & $\begin{array}{c}200 \\
\text { ppm }\end{array}$ & $\begin{array}{c}400 \\
\text { ppm }\end{array}$ & $\begin{array}{c}800 \\
\text { ppm }\end{array}$ & $\begin{array}{l}1000 \\
\text { ppm }\end{array}$ & $\begin{array}{l}2000 \\
\text { ppm }\end{array}$ & Control & Mean \\
\hline 1. & $15 \mathrm{~cm}$ soft wood cutting + IAA & 14.33 & 13.00 & 11.66 & 11.00 & 10.33 & 0.00 & 10.05 \\
\hline 2. & $\begin{array}{l}15 \mathrm{~cm} \text { semi hard wood Cutting + } \\
\text { IAA }\end{array}$ & 8.33 & 9.66 & 10.33 & 7.66 & 7.33 & 7.33 & 8.44 \\
\hline 3. & $15 \mathrm{~cm}$ hard wood cutting + IAA & 9.66 & 11.00 & 10.33 & 8.66 & 11.00 & 8.66 & 9.88 \\
\hline 4. & $15 \mathrm{~cm}$ soft wood cutting + IBA & 16.00 & 19.66 & 20.00 & 24.33 & 17.33 & 0.00 & 16.22 \\
\hline 5. & $\begin{array}{l}15 \mathrm{~cm} \text { semi hard wood Cutting }+ \\
\text { IBA }\end{array}$ & 8.66 & 9.33 & 10.66 & 10.00 & 8.66 & 7.33 & 9.11 \\
\hline 6. & $15 \mathrm{~cm}$ hard wood cutting + IBA & 13.33 & 17.66 & 16.66 & 19.66 & 15.00 & 8.66 & 15.16 \\
\hline 7. & $25 \mathrm{~cm}$ soft wood cutting + IAA & 11.00 & 11.00 & 11.66 & 14.33 & 15.66 & 9.00 & 12.11 \\
\hline 8. & $\begin{array}{l}25 \mathrm{~cm} \text { semi hard wood Cutting + } \\
\text { IAA }\end{array}$ & 5.33 & 5.66 & 9.33 & 10.33 & 12.33 & 6.33 & 8.22 \\
\hline 9. & $25 \mathrm{~cm}$ hard wood cutting + IAA & 9.00 & 11.33 & 13.00 & 13.33 & 24.66 & 8.00 & 13.22 \\
\hline 10. & $25 \mathrm{~cm}$ soft wood cutting + IBA & 9.00 & 10.33 & 12.66 & 16.33 & 8.00 & 9.00 & 10.88 \\
\hline 11. & $\begin{array}{l}25 \mathrm{~cm} \text { semi hard wood Cutting }+ \\
\text { IBA }\end{array}$ & 13.33 & 10.00 & 9.00 & 4.00 & 12.00 & 6.33 & 9.11 \\
\hline \multirow[t]{2}{*}{12} & $25 \mathrm{~cm}$ hard wood cutting + IBA & 21.33 & 6.00 & 23.00 & 10.66 & 15.66 & 8.00 & 14.11 \\
\hline & Mean & 11.61 & 11.22 & 13.19 & 12.52 & 13.16 & 6.88 & \\
\hline
\end{tabular}

Cutting type - CD at 5\% -1.90; Concentration - CD at 5\%- 1.34; Interaction CD at 5\% -4.66

Table 5. Effect of IAA and IBA on fresh biomass $(\mathrm{g})$ in different types of cuttings of $P$. pinnata.

\begin{tabular}{|c|c|c|c|c|c|c|c|c|}
\hline $\begin{array}{l}\text { S. } \\
\text { N. }\end{array}$ & Cutting Type & $\begin{array}{l}200 \\
\text { ppm }\end{array}$ & $\begin{array}{l}400 \\
\text { ppm }\end{array}$ & $\begin{array}{l}\mathbf{8 0 0} \\
\text { ppm }\end{array}$ & $\begin{array}{l}1000 \\
\text { ppm }\end{array}$ & $\begin{array}{l}2000 \\
\text { ppm }\end{array}$ & Control & Mean \\
\hline 1. & $15 \mathrm{~cm}$ soft wood cutting + IAA & 14.00 & 10.66 & 11.66 & 13.33 & 14.33 & 0.00 & 10.66 \\
\hline 2. & $\begin{array}{l}15 \mathrm{~cm} \text { semi hard wood Cutting }+ \\
\text { IAA }\end{array}$ & 15.66 & 17.00 & 17.66 & 20.00 & 17.33 & 11.33 & 16.50 \\
\hline 3. & $15 \mathrm{~cm}$ hard wood cutting + IAA & 27.66 & 32.00 & 29.00 & 26.00 & 32.33 & 21.33 & 28.05 \\
\hline 4. & $15 \mathrm{~cm}$ soft wood cutting + IBA & 13.00 & 10.00 & 11.00 & 12.33 & 14.33 & 0.00 & 10.11 \\
\hline 5. & $\begin{array}{l}15 \mathrm{~cm} \text { semi hard wood Cutting + } \\
\text { IBA }\end{array}$ & 13.33 & 15.00 & 15.66 & 15.66 & 17.33 & 11.33 & 14.71 \\
\hline 6. & $15 \mathrm{~cm}$ hard wood cutting + IBA & 47.00 & 31.00 & 29.33 & 32.66 & 36.66 & 21.33 & 33.00 \\
\hline 7. & $25 \mathrm{~cm}$ soft wood cutting + IAA & 20.33 & 22.33 & 26.66 & 42.66 & 40.00 & 19.66 & 28.61 \\
\hline 8. & $\begin{array}{l}25 \mathrm{~cm} \text { semi hard wood Cutting }+ \\
\text { IAA }\end{array}$ & 9.33 & 20.00 & 24.00 & 23.00 & 27.00 & 20.33 & 20.61 \\
\hline 9. & $25 \mathrm{~cm}$ hard wood cutting + IAA & 20.33 & 22.00 & 31.33 & 51.33 & 49.66 & 20.00 & 32.44 \\
\hline 10. & $25 \mathrm{~cm}$ soft wood cutting + IBA & 20.00 & 25.66 & 27.00 & 50.66 & 35.66 & 19.66 & 29.77 \\
\hline 11. & $\begin{array}{l}25 \mathrm{~cm} \text { semi hard wood Cutting + } \\
\text { IBA }\end{array}$ & 63.66 & 52.66 & 39.33 & 44.00 & 43.66 & 20.33 & 43.94 \\
\hline \multirow[t]{2}{*}{12} & $25 \mathrm{~cm}$ hard wood cutting + IBA & 24.00 & 29.00 & 30.33 & 31.66 & 35.00 & 20.00 & 28.33 \\
\hline & Mean & 24.02 & 23.94 & 24.417 & 30.27 & 30.27 & 15.44 & \\
\hline
\end{tabular}

Cutting type - CD at 5\% - 2.87; Concentration - CD at 5\% - 2.03; Interaction CD at 5\%-7.04

IAA which may be due to the higher concentration of IAA and or synergic effect of indigenous and exogenous auxin (IAA) applied on the cuttings making favorable condition for producing maximum root number. Negi and Tiwari (1984) also reported that higher concentration of some growth hormones promoted maximum number of roots in $P$. pinnata . This may be also due to enhanced hydrolysis of carbohydrates caused by auxins treatments (Rajarama, 1997).In case of soft wood, semi hard wood and hard wood cuttings of $15 \mathrm{~cm}$ and $25 \mathrm{~cm}$ length treated with IBA $1000 \mathrm{ppm}$ and 400 ppm IAA respectively resulted in maximum root number. However in case of soft wood, semi hard wood and hard wood cuttings of $25 \mathrm{~cm}$ length treated with IAA $2000 \mathrm{ppm}$ and IBA $800 \mathrm{ppm}$ respectively resulted in maximum root number. This may be due to higher concentration of IBA are also beneficial for promotion of rate of rooting and heavier root system in stem cutting of $P$. pinnata ( Negi and Tiwari ,1984.). The result of fresh biomass (Table 5) observed was maximum (43.94 g) in semi hard wood cuttings of $25 \mathrm{~cm}$ length treated with IBA, However maximum fresh biomass $(63.66 \mathrm{~g})$ was recorded from semi hard wood cuttings of $25 \mathrm{~cm}$ length treated with $200 \mathrm{ppm}$ IBA. The possible reason for this may be IBA treatment enhances the growth of cutting raised 
Table 6. Effect of IAA and IBA on dry biomass (g) in different types of cuttings of P. pinnata.

\begin{tabular}{|c|c|c|c|c|c|c|c|c|}
\hline S. $\mathbf{N}$. & Cutting Type & $\begin{array}{c}200 \\
\text { ppm }\end{array}$ & $\begin{array}{c}400 \\
\mathrm{ppm}\end{array}$ & $\begin{array}{c}800 \\
\text { ppm }\end{array}$ & $\begin{array}{l}1000 \\
\text { ppm }\end{array}$ & $\begin{array}{l}2000 \\
\text { ppm }\end{array}$ & Control & Mean \\
\hline 1. & $15 \mathrm{~cm}$ soft wood cutting + IAA & 5.52 & 3.40 & 3.38 & 5.06 & 5.75 & 0.00 & 3.85 \\
\hline 2. & $\begin{array}{l}15 \mathrm{~cm} \text { semi hard wood Cutting + } \\
\text { IAA }\end{array}$ & 6.13 & 5.99 & 6.36 & 6.90 & 6.17 & 5.76 & 6.22 \\
\hline 3. & $15 \mathrm{~cm}$ hard wood cutting + IAA & 12.86 & 14.74 & 12.03 & 11.24 & 14.89 & 7.46 & 12.20 \\
\hline 4. & $15 \mathrm{~cm}$ soft wood cutting + IBA & 5.53 & 2.97 & 3.68 & 3.29 & 4.00 & 0.00 & 3.24 \\
\hline 5. & $\begin{array}{l}15 \mathrm{~cm} \text { semi hard wood Cutting }+ \\
\text { IBA }\end{array}$ & 6.15 & 6.73 & 6.70 & 7.00 & 7.39 & 5.76 & 6.62 \\
\hline 6. & $15 \mathrm{~cm}$ hard wood cutting + IBA & 18.06 & 14.00 & 13.63 & 15.37 & 17.80 & 7.46 & 14.39 \\
\hline 7. & $25 \mathrm{~cm}$ soft wood cutting + IAA & 9.06 & 9.03 & 10.83 & 14.73 & 14.17 & 9.71 & 11.25 \\
\hline 8. & $\begin{array}{l}25 \mathrm{~cm} \text { semi hard wood Cutting }+ \\
\text { IAA }\end{array}$ & 7.83 & 10.06 & 9.65 & 8.81 & 10.10 & 8.48 & 9.15 \\
\hline 9. & $25 \mathrm{~cm}$ hard wood cutting + IAA & 9.12 & 11.33 & 13.89 & 34.38 & 30.88 & 15.17 & 19.12 \\
\hline 10. & $25 \mathrm{~cm}$ soft wood cutting + IBA & 9.66 & 11.02 & 12.25 & 24.94 & 14.86 & 9.71 & 13.74 \\
\hline 11. & $\begin{array}{l}25 \mathrm{~cm} \text { semi hard wood Cutting }+ \\
\text { IBA }\end{array}$ & 18.59 & 21.83 & 17.38 & 18.39 & 15.71 & 8.48 & 16.73 \\
\hline \multirow[t]{2}{*}{12} & $25 \mathrm{~cm}$ hard wood cutting + IBA & 17.02 & 19.34 & 16.37 & 17.03 & 16.79 & 15.17 & 16.95 \\
\hline & Mean & 10.46 & 10.87 & 10.51 & 13.93 & 13.21 & 7.76 & \\
\hline
\end{tabular}

Cutting type $-\mathrm{CD}$ at 5\% - 4.89; Concentration $-\mathrm{CD}$ at 5\%-3.46; Interaction CD at 5\%-12.00

plants because of more root numbers, root length and faster cambial activity than the hard wood and soft wood cuttings. The higher food resources in large cuttings could be another reason for their better growth and development. Zhang et al. (2010) reported a significant increase in root length and root biomass and shoot length of cuttings with increase in diameter of cuttings in Feijoa sellowiana. IBA treatment enhances the cambial activity (Davies et al., 1982; Davies, 1984).In case of soft wood, semi hard wood and hard wood cuttings of $15 \mathrm{~cm}$ length treated with IAA 2000 ppm, 1000 ppm and IBA 200 ppm, respectively resulted in maximum fresh biomass. However incase of soft wood, semi hard wood and hard wood cuttings of $25 \mathrm{~cm}$ length treated with 1000 ppm IAA and 1000 ppm and 200 ppm, IBA respectively resulted in maximum fresh biomass. Similarly for the dry biomass it observed maximum (19.12 g) in hard wood cuttings of $25 \mathrm{~cm}$ length treated with IAA $1000 \mathrm{ppm}$. The highest biomass obtained in $25 \mathrm{~cm}$ hard wood cuttings treated with IAA (34.38g) (Table 6 ). This may be due to growth hormone determine cell elongation and cell division there by promoting root length (Abidin and Baker,1984) and consequently resulting in better growth of the cutting this might be the result in increasing the dry biomass. The dry biomass was more in treated hard wood cuttings raised plants than the treated semi hard wood cuttings raised plants (which shows maximum fresh biomass) may be because hard wood cuttings raised plants have less fresh biomass resulting in less moisture content in shoot portion than the semi hard wood raised plants. In case of soft wood, semi hard wood and hard wood cuttings of $15 \mathrm{~cm}$ length treated with IAA $2000 \mathrm{ppm}$ and IBA 200ppm, $2000 \mathrm{ppm}$ respectively resulted in maximum fresh bio- mass. However incase of soft wood, semi hard wood and hard wood cuttings of $25 \mathrm{~cm}$ length treated with 1000 ppm IAA and 400 ppm IBA respectively resulted in maximum fresh biomass.

\section{Conclusion}

It was concluded that the Hard wood cuttings of $25 \mathrm{~cm}$ length from plus tree of P.pinnata produces seedlings when treated with IBA promotes better sprouting, rooting percent $(90 \%)$, root number and biomass than IAA. For production of healthy seedlings cutting of $25 \mathrm{~cm}$ length should be treated with IBA. Hence this treatment may be preferred over other treatments for vegetative propagation through stem cuttings of $P$. pinnata for mass multiplication.

\section{REFERENCES}

Abidin,M. and Baker,A.(1984).Effect of growth substances on rooting of Cocoa cuttings. Acta Hortculture, 137:95-102.

Brijesh, S., Daswani, P.G., Tetali, P., Rojatkar, S.R., Anita, N.H. and Birdi, T.J. (2006). Studies on Pongamia pinnata (L.) Pierre leaves: understanding the mechanism(s) of action in infectious diarrhea. Journal of Zhejiang University SCIENCE B, 7: 665-74.

Chandramouli, H. (2001). Influence of growth regulators on the rooting of different types of cuttings in Bursera penicilliatai (DC) Engl. M.Sc. ( Agril )Thesis, Univ. Agric. Sci. Bangalore.

Chaturvedi, M.D. (1975). The common Karanja. Indian Farming, 7(2): 8- 9.

CSIR. (1966). The Wealth of India: raw materials. Vol. 110. Council of Scientific and Industrial Research (CSIR), New Delhi, India 1948-98.

Davies, F.T. (1984).Shoot RNA, cambial activity and Indole butyric acid effectivity in seasonal rooting of juvenile and mature Ficus pumila cuttings.Physiol.Plant., 62:571 -575 . 
Davies, F.T., Lazarate, J.E., and Joiner, J.N. (1982).Initiation and development of roots in juvenile and mature leaf bud cuttings of Ficus pumila L.Am.J.Bot., 69: 804-811.

Debnath, G.C. and Maiti, S.C. (1990). Effect of the growth regulators on rooting of softwood cuttings of guava (Psidium guajava L.) under mist. Haryana .J. Hort. Sci., $19: 79-85$.

De Klerk, G.,Terbrugge,J.Maronova,S. (1997). Effectiveness of IAA,IBA and NAA during adventitious roots formation in vitro in ,alus 'Jork 9'.Plant cell Tissue organ culture, $49: 39-44$.

Epstein, E., Ludwig-Muller, J. (1993). Indole-3-butyric acid in plants occurance, synthesis, metabolism and transport. Physiol. Plant, $88: 382-389$.

Ghatnatti, S.A. (1997). Studies on propagation of Durantha plumeri Jacq. Var Goldiana by stem cuttings with growth regulators under mist. M.Sc.(Agri.) Thesis, Univ. Agric. Sci., Dharwad (India).

Gomez, K.A. and Gomez, A.A. (1984). Statistical procedures for Agriculture Research ( $2^{\text {nd }} e d n$.) John Willey and Sons, Inc., New York. 68 p.

Hartmann, H. T., Kester, D. E., Davies, F. T., Geneve, R.L. (2002). Hartmann and Kester's Plant Propagation: Principles and practices $.7^{\text {th }}$ ed. Prentice Hall, Upper saddle River, pp 298-342

Haissig, B.E. (1984).Carbohydrate accumulation and partitioning in Pinus banksiana seedlings and seedling cuttings. Physiologia plantarum, 61:13-19.

Hewamanna, R., Anuraadhai, N. and Fernando, R.K.S. (2004). Analysis of five trace elements in medicinal plants used in Ayurvedic medicine to control diabetes. Journal of Tropical medicinal plants, 5 (2): 211-215.

Kesari,V., A. Das, and L. Rangan (2008). Physico-chemical characterization and antimicrobial activity from seed oil of Pongamia pinnata, a potential biofuel crop. Biomass and Bioenergy, 34 (1):108.

Krisantini,S., Johnston, M.,Williams, R.R., Beveridge, L. (2006). Adventitious root formation in Grevillea ( Proteaceae) an Australian native species. Sci Hortic., 107:171-175.

Mandal, L. and Banarjee, G.C. (1982). Studies on the utilization of Karanja (Pongamia glabra Vent.) cake in polutary rations effects on growers and in blood composition and organ weights of Cockerels. Indian Veterinary Journal, 59 (5) :385-390.

Mullins, M.G. (1972). Auxin and ethylene in adventitious root formation in Phaseolus aureus (Roxb.)In: Carr DJ ed. Plant growth substances Springer, New York. pp 526-533.
Nanda, K. K., Purohit, A.N. and Mehrotra, K. (1968). Effect of sucrose, auxins and gibberellic acid on rooting of stem segments of Poplus nigra under verifying light condition. Plant and Cell Physiology, 9:735-743.

Negi, K.S. and Tiwari, C.K.(1984).Vegetative propagation in cuttings of Pongamia pinnata L.Pierre by auxin Reg.For.Res.Centre. Jabalpur. 110 (7):655-659.

Palanisamy, K. and Kumar, P. (1997). Seasonal variation on Adventitious rooting in branch cuttings of Pongamia pinnata (Pierre). Indian Forestor, 122(3):236-239.

Rahman, A.H.M.M. (1977). Vegetative propagation of few Forest species. Bano Vigyan Patrika, 6 (1) : 51-57.

Rajarama, H.A. (1997). Studies on propagation of Pomegranate (Punica granatum L.) by cuttings. M.Sc. (Agri.) Thesis, Univ. Agric. Sci., Dharwad.

Sahoo, P. K., Behera, L.K. and Nayak, S. (2014). Vegetative propagation of Physic nut (Jatopha curcas L.) through stem cuttings. Journal of Applied and Natural Science, 6 (2):467-472.

Shagoo, P., Beigh, M. A., Lone, R.A. and Nanda, A.B, (2007). Effect of plant growth regulators on rooting of Barbados cherry. Asian Journal of Horticulture, 2 (1):152-154.

Smith, N.G. and Wareing, P.E. (1972). The rooting of actively growing and dormant leafy cuttings in relation to endogenous hormone levels and photoperiod. New Phyto., 71:483-500.

Strydom, D.K., Hartmann, H.T. (1960). Absorption, Distribution, and Destruction of Indole acetic Acid in Plum Stem Cuttings. Plant Physiol., 35 (4) : 435-442.

Troup, R.S. (1921). The silviculture of Indian trees. Clarendon Press, Oxford, UK.

Waisel,Y. and Fahn,A.(1965).The effect of environment on wood formation and cambial activity on Robinia pseudacacia L. New Phytol., 64:346-442.

Wareing,P.F. and Roberts, D.L. (1956). Photoperiodic control of cambial activity in Robinia pseudacacia. New Phytol., 55 : 356-366.

Wright, R.C.M. (1975). The complete hand book of Plant Propagation .Macmillan, New York.

Zerhoune, M. (2003). Propagation of Five Fig (Ficus Carica L.) varieties under field conditions. Acta Horticulturae, 605:103-106.

Zhang, M., Wang, D., Ren, S., Fan, L. and Lia, R. (2010). Effects of Feijoa diameter on seedling quality. Agril. Journal, 5(5) : 139-141. 